\title{
DEVICE ENABLED ARCHITECTURE FOR CONTEXT AWARE APPLICATIONS
}

\author{
G K Mohan Devarakonda \\ Department of Computer Science \\ Krishna University, Machilipatnam, \\ Andhra Pradesh, India
}

\author{
Y. K. Sundara Krishna \\ Department of Computer Science \\ Krishna University Machilipatnam, India
}

\begin{abstract}
Context awareness, a key capability of ubiquitous computing is spreading in a variety of applications includes desktop applications, web applications, mobile applications and Internet of Things. It is mandatory in most of the context aware applications to determine the location of user to provide the service. The service is solely based on user location and the availability of service. This paper proposes a new architecture for context aware applications so that users can avail the services based on a variety of context information in a more convenient manner according to their requirement.
\end{abstract}

Keywords: context awareness, user context, context information

\section{INTRODUCTION}

The concept of context awareness is raised in the early 1990s; however it became popular with the introduction of the term ubiquitous computing [1]. The context aware applications users are availing

the service based on their location which is determined using Global Positioning System (G.P.S) or Geographical Information Systems (G.I.S).

Integration of heterogeneous systems such as electronic, electromagnetic, optical and sensor devices to the World Wide Web leads to the generation of several types of information in offline and online mode. These devices in the availability of context information further leads to the development of varieties of context aware applications. The integration of hardware, software and networking components give rise to development of a novel architecture for context

based systems and coins a new technology called context aware computing. Most of the systems and solutions that are developed are application specific and sometimes requirement specific and belongs to a business domain.

These types of services are mostly based on the user location information which includes place, time and geographical location and providing various services such as tracking, navigation, Advertising, Communication and Social Services ${ }^{[3]}$. The user mobile device will provide its location information to the context server where the entire database for the context is maintained. The context server then performs location mapping, service mapping and other related operations on data acquired from user mobile to decide services that a user can avail which includes processing by the context server.

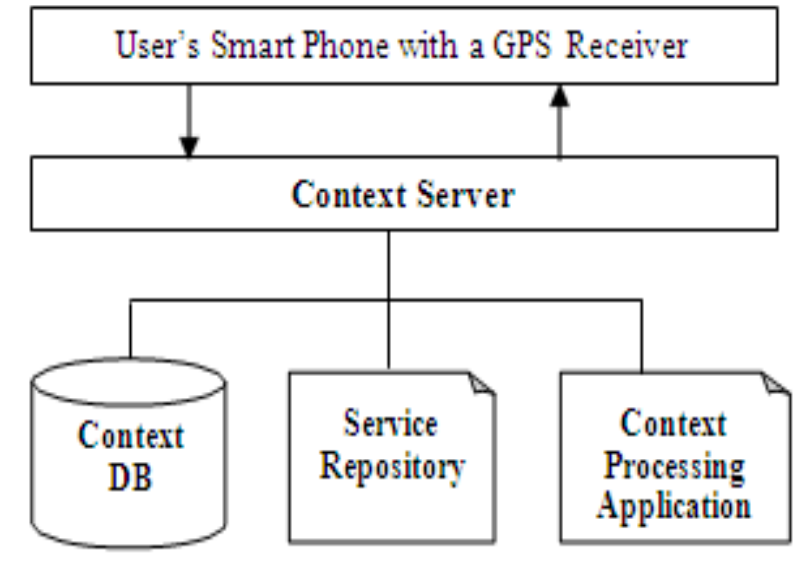

fig. 1 Architecture of Context Aware Applications

The processing of user request for a context aware service is solely done by the context server only after collecting the data from user, context database and service repository ${ }^{[4]}$. As the service is utilized by a large number of users at any time, any place and any location, the context server will experience a lot of processing burden. This causes service discrepancies and makes the service unavailable when large number of user requests hit the context server for a service. This paper proposes a new architecture for context aware applications to efficiently manage the context data and to provide faster service in a variety of contexts to the user by reducing the processing burden on the server.

\section{PROPOSED SYSTEM}

The proposed context aware system is equipped with a device called Context Information Device which is configurable dynamically pertaining to any context. This device is installed at the location where the particular service is provided. On the other hand, the user will avail the services using his mobile device which is context enabled. The context enabled mobile device of the user also has an application that allows the user to configure his requirement (User Context) through context app. When the user enters 
the region of a particular context information device, the device will detect the user device. The user device will send its context information to the context information device through an interface provided. The context information device will map its context data with that of user context by applying suitable algorithms to verify available services to the particular user and to provide service accordingly. Information in the both the context enabled mobile device (User Context) and context information device (Service Context) are configurable dynamically and are designed using suitable data structures.

\section{ARCHITECTURE}

The proposed architecture for context aware applications namely, Device Enabled Architecture for Context Aware Applications will consists of the components namely Context Information Device, Context Enabled Mobile Device and an Interface. Proper utilization and maintenance of Context Information device will help the user to identify the available context around him. The context app of user mobile device of user is able to receive signals from context information device and also the user can configure his requirement in this app.

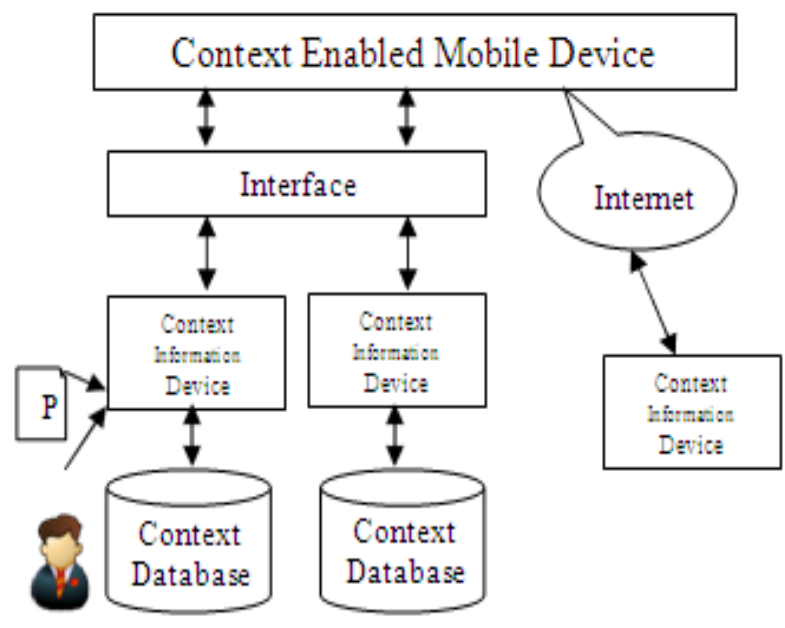

\section{Fig. 2 Device Enabled Architecture for Context Aware Applications}

\section{Context Enable Mobile Device:}

This device is a user smart phone which is equipped with context app to send and receive information and services from the context information device. The context app in this device allows the user to configure their requirements and consists of suitable data structures that can be configured dynamically.

\section{Context Information Device:}

Context Information Devices are configured according to their contexts using the dynamically configurable data structure and placed in their respective locations where the service to the user is provided in the respective context. The information in these devices is dynamically configurable through its administrator or various programs or even from the internet sources.

\section{Context Database}

This is the database where the information about a particular service is completely available. The database frequently configures the context information device with an updated data and service structures.

Whenever a user asks for a context, the context enabled mobile device of user will provide the user requirement configured in itself to the context information device. The information in the context enabled mobile device is mapped with the information in context information device which is already aware of all the services that are available in that region. This mapping is done using suitable algorithms and methodologies and finally it is concluded that whether the context information device context matches the user required context. If it matches the user requirement, the user then can avail the service based on his location which is determined by using wither GPS or GIS, otherwise he may continue in search of another context.

For example, a user who is travelling may configure his context enabled mobile device with his travel details such as travel mode (ex: train) and time requirements. Whenever the user enters in the premises of the source station, the context information device in the source station detects the user device and the requirements of the user, and maps the user context with its own context and provides the user with relevant information services such as available train number, arrival time and departure time etc..

Availing the services from the context information device doesn't restrict the user from being connected to the regular internet and other services. In fact, the context information devices are also configured in such a way that they can provide their services through the internet even.

In both the context information device and the context app in user mobile device will maintain context information in two categories i.e., Static Context Information and Dynamic Context Information. Information that is unlikely to change frequently i.e., constant data is represented in static context information and the information that has a chance to change quiet frequently is represented with dynamic context information and the mapping is done with respective context information.

In earlier architecture of context aware applications, the work flow suffers from the processing burden on the server. But in this proposed architecture there will not be any processing burden because every context related activity is processed in a context information device separately for each context.

\section{ADVANTAGES}

- This architecture eliminates the concept of having a central server as the entire information processing is done by the context information device for every context.

- User's need not maintain internet connection throughout the service there by significant amount of user's mobile data is saved.

- User can avail the services as per his requirement based on his own requirement.

- No need to determine user location until unless user decides to use the service.

- This methodology will work as a virtual publicity for business services.

\section{CONCLUSION}

The context aware applications are becoming popular with 
variety of services attracting huge number of user's day-byday. One can concentrate on the performance of the context aware applications to provide the services in amore convenient and effective manner. The Device Enabled Architecture for Context Aware Applications helps to provide services to the user in an effective manner with the minimal intervention of internet and World Wide Web.

\section{REFERENCES}

[1] Charith Perera, Arkady Zaslavsky, and Dimitrios Georgakopoulos, “Context Aware Computing for The
Internet of Things : A Survey" IEEE Communication Surveys \& Tutorials, vol.16,Issue 1, First Quarter 2014.

[2] Carnot Institutes, "Smart networked objects and Internet of Things", Carnot institutes Information communication technologies and Micro Nano technologies alliance, White Paper, January,2011.

[3] Matthias Baldauf, "A survey on context-aware systems", Internation Journal of Adhoc and Ubiquitous Computing Vol.2 No.4 2007.

[4] Bin Jiang, Xiao bai Yao, "Location based Services and GIS in perspective”, Computer environments and subsystems, Elsevier 2016. 